Supporting Information

\title{
Recognition, intervention, and monitoring of neutrophils in acute ischemic stroke
}

Chunming Tang, Cong Wang, Ying Zhang, Lingjing Xue, Yanyi Li, Caoyun Ju*, and Can Zhang*

State Key Laboratory of Natural Medicines and Jiangsu Key Laboratory of Drug

Discovery for Metabolic Diseases, Center of Advanced Pharmaceuticals and Biomaterials, China Pharmaceutical University, Nanjing 210009, P. R. China

*E-mail: zhangcan@cpu.edu.cn. Phone/Fax number: 86-25-83271171

*E-mail: jucaoyun@cpu.edu.cn. Phone number: 86-25-83271076 
Mice. ICR mice (male, 6-8 weeks old) and C57BL/6 mice (male, 6-8 weeks old) were purchased from the Comparative Medicine Center of Yangzhou University. All the animal experiments were performed in compliance with the Guide for Care and Use of Laboratory Animals approved by China Pharmaceutical University.

The mouse transient middle cerebral artery occlusion (tMCAO) model was prepared using a modified intraluminal filament technique. ${ }^{1}$ Briefly, the right common carotid artery (CCA), external carotid artery (ECA) and internal carotid artery (ICA) were carefully dissected and exposed through midline neck incision. MCA occlusion was performed by introducing a silicon-coated 6-0 monofilament suture (Doccol Corporation) into the CCA through ECA and advancing it $9 \pm 1 \mathrm{~mm}$ via ICA to the origin of MCA. Reperfusion was initiated by withdrawing the filament after $1 \mathrm{~h}$. Apart from sham group, all other groups were performed MCAO/reperfusion surgery. All the groups were performed and quantified in a randomized fashion by investigators blinded to treatment groups.

Synthesis and characterization of SPIO. The monodisperse SPIO nanoparticles were synthesized by using a one-pot hydrothermal method. ${ }^{2}$ Briefly, 2 mmol of $\mathrm{Fe}(\mathrm{acac})_{3}$ was dissolved in a mixture of $10 \mathrm{ml}$ benzyl ether and $10 \mathrm{~mL}$ oleylamine. The solution was dehydrated at $110{ }^{\circ} \mathrm{C}$ for $1 \mathrm{~h}$, and was quickly heated to $300{ }^{\circ} \mathrm{C}$ and kept at this temperature for $2 \mathrm{~h} .50 \mathrm{~mL}$ of ethanol was added into the solution after it was cooled to room temperature. The precipitate was collected by centrifugation at $4500 \mathrm{rpm}$ and was washed with ethanol 3 times. The final product SPIO nanoparticles 
were re-dispersed in dichloromethane for future use. Samples for TEM were prepared by drying the obtained SPIO nanoparticle solutions on copper grid and imaged by a transmission electron microscopy (TEM, Hitachi H-7650) at $80 \mathrm{kV}$. X-ray powder diffraction patterns of the obtained SPIO nanoparticle were measured on a Bruker AXS D8-Advanced diffractometer with $\mathrm{Cu} \mathrm{K \alpha}$ radiation $(\lambda=1.5418 \AA)$. Magnetic properties were studied using a Lakeshore 7300 high sensitivity vibrating sample magnetometer (VSM) with fields up to 1.5 tesla at room temperature. The particles were mixed with $\mathrm{KBr}$ powder and then pressed into pellets at 16000 psi. A Nicolet Nexus 870 fourier transform infrared spectrometer was used for FT-IR spectrum recording.

Preparation and characterization of PTNPs. PLGA nanoparticles containing SPIO nanocrystals and piceatannol were prepared using a modified conventional oil-in-water $(\mathrm{O} / \mathrm{W})$ single emulsion evaporation method. ${ }^{3}$ Briefly, $10 \mathrm{mg}$ PLGA and 3 mg SPIO nanocrystals were added to $800 \mu \mathrm{L}$ methylene chloride, and $0.5 \mathrm{mg}$ piceatannol was dissolved in $200 \mu \mathrm{L}$ acetone. This mixture was emulsified with 10 $\mathrm{mL}$ of the PVA aqueous solution ( $1 \mathrm{wt} \%$ ) using a probe type sonicator at $100 \mathrm{w}$ for 5 min in ice bath. The resulting suspension was stirred for $6 \mathrm{~h}$ at room temperature to evaporate organic solvent and was subsequently centrifuged at $13000 \mathrm{rpm}$ for $30 \mathrm{~min}$. The obtained TNPs were washed three times with distilled water and resuspended in water. The Cy5 and Rhodamine labeled PLGA nanoparticles were prepared with the 
similar method described above except using $0.1 \mathrm{wt} \%$ Cy5 instead of SPIO nanocrystals and piceatannol or PLGA- Rhodamine instead of PLGA-COOH.

In order to prepare platelet membrane-cloaked TNPs, Platelets from whole blood were isolated through gradient centrifugation. ${ }^{4}$ Briefly, the whole mouse blood was centrifuged at $300 \mathrm{~g}$ for $3 \mathrm{~min}$ at room temperature. The supernatant was then collected and spun at the same speed for another $3 \mathrm{~min}$. Afterwards, the resulting supernatant was centrifuged at $800 \mathrm{~g}$ for $4 \mathrm{~min}$ to pellet down the platelets. The platelets were washed by the PBS buffer and centrifuged repeatedly. The purified platelets were resuspended in water and stored at $-80{ }^{\circ} \mathrm{C}$ for further use. Platelet membrane was derived by a repeated freeze-thaw process. Aliquots of platelet suspensions were firstly frozen at $-80{ }^{\circ} \mathrm{C}$, thawed at room temperature and pelleted by centrifugation at $16000 \mathrm{~g}$ for $5 \mathrm{~min}$, followed by resuspending in water. The platelet suspension was refrozen, and the process was repeated three times. The obtained platelet membranes were suspended in water and sonicated in a capped glass vial for 5 min using a bath sonicator. Finally, platelet membrane cloaking was accomplished by dispersing and fusing platelet membrane vesicles with TNPs nanoparticles by sonication using a bath sonicator for another $5 \mathrm{~min}$. The particle size and surface zeta potential of platelet vesicles, TNPs and PTNPs were characterized using DLS, respectively. The morphologies were determined by a transmission electron microscopy (TEM, JEM-200CX, JEOL, Japan). The samples were prepared by contacting the nanoparticle droplets at a concentration of $100 \mu \mathrm{g} / \mathrm{mL}$ with copper grids for $5 \mathrm{~min}$, removing the excess droplets, and staining by $1 \%$ uranyl acetate for 
$30 \mathrm{~s}$ before the TEM studies. Loading efficiency of piceatannol and $\mathrm{Fe}_{3} \mathrm{O}_{4}$ nanocrystals were quantified by high performance liquid chromatography (HPLC, LC-2010AHT, SHIMADZU, Japan) and ICP-AES (Optima 5300DV, PE, USA).

To optimize the platelet membrane to TNPs core ratio, different amount of platelet membrane was incubated with the TNPs at membrane protein-to-core weight ratio ranging from 0.05 to 2 . The amount of proteins retained on the obtained PTNPs were determined by BCA assay. The membrane coating efficiency of SPIO-PTNPs and PTNPs was determined by ICP-AES.

The integrity of the PTNPs was determined by the co-localization of the membrane and the cores. Mature neutrophils were isolated from murine bone marrow using a modified method. ${ }^{5}$ Briefly, the femur and the tibia from both hind legs were immersed in RPMI 1640 medium after removal of the muscle and sinew. Bone marrow was flushed from the bone with HBSS-EDTA solution, centrifuged at $200 \mathrm{~g}$ for $3 \mathrm{~min}$ and resuspended in $1 \mathrm{~mL}$ HBSS-EDTA. The cells were laid on a Percoll mixture solution consisting of $55 \%, 65 \%$ and $75 \%$ Percoll, followed by centrifugation at $1500 \mathrm{~g}$ for 30 $\min$. The mature neutrophils were recovered at the interface of the $65 \%$ and $75 \%$ fractions and washed by ice-cold HBSS-EDTA thrice. The purity was determined by Alexa Fluor 488-labeled anti-Gr-1 antibody (BioLegend, San Diego, CA). The morphology of neutrophils was determined by staining with Wright-Giemsa (Jiancheng Bio, China). Dual-labeled PTNPs, Rhodamine labeled TNP core and DiO labeled membrane, were incubated with mouse bone marrow neutrophils for $30 \mathrm{~min}$. Hoechst 33342 was used to stain the nuclei for $15 \mathrm{~min}$. Subsequently, the cells were 
washed with PBS and fixed with 4\% paraformaldehyde for 15 min before imaging under a confocal laser scanning microscope (CLSM, LSM 880, Zeiss).

The in vitro stability of TNPs and PTNPs were evaluated by measuring the diameter in $100 \%$ FBS for $24 \mathrm{~h}$ and in $1 \times$ PBS for $7 \mathrm{~d}$ using DLS.

For in vitro piceatannol release, $1 \mathrm{~mL}$ of PTNPs or mixture of $0.5 \mathrm{~mL}$ PTNPs and $0.5 \mathrm{~mL}$ FBS were added into a dialysis tube (3.5K MWCO) embedded into $50 \mathrm{~mL}$ of the PBS buffer solution $(\mathrm{pH}=7.4)$ containing Tween $80(1 \%, \mathrm{w}: \mathrm{v})$, and gently shaken at $37^{\circ} \mathrm{C}$ in a shaker at $100 \mathrm{rpm}$. At predetermined time intervals, $1 \mathrm{~mL}$ of the medium was drawn out and replaced with an equal volume of fresh medium. Then, piceatannol was extracted and the piceatannol concentration was determined by HPLC.

Coomassie blue staining and Western blotting analysis were employed to identify the proteins on platelets, platelet membranes vesicles and PTNPs. Briefly, the samples containing equivalent total proteins measured by BCA protein assay kit (Life Technologies) were added to the $10 \%$ SDS-polyacrylamide gel to separate different molecular weights of proteins. Subsequently, the resulting polyacrylamide gel was stained with Commassie blue and imaged. The key membrane proteins were identified by western blotting using primary antibodies including anti-CD47 (absin, China), anti-CD41 (Abcam, Cambridge, MA), and anti-CD62P (Abcam, Cambridge, MA).

The MRI imaging capability of SPIO-TNPs, TNPs, and PTNPs were evaluated by using a 7.0 T whole body MRI system (Biospec 7T/20 USR, Bruker, Germany). $T_{2}$-weighted MRI imaging of SPIO-TNPs, TNPs, and PTNPs at a series of Fe concentrations were acquired for $T_{2}$ relaxation rate $\left(r_{2}\right)$ assessment. 
In vitro immune escape evaluation. RAW 264.7 cells $\left(1 \times 10^{5}\right.$ cells/per dish or well $)$ were seeded in confocal dishes and 6-well plates, and cultured for $24 \mathrm{~h}$. Then the cells were incubated with Rhodamine-labeled TNPs and PTNPs and incubated for $4 \mathrm{~h}$. Thereafter, the cells in confocal dishes were washed with PBS for three times, stained with Hoechst 33342, fixed with 4\% PFA and imaged by CLSM (LSM 880, Zeiss). To quantify Fe uptake by RAW 264.7 cells, the cells in 6-well plates were lysed by adding $0.5 \mathrm{~mL} 1 \%$ Tween 80 to each well. The cell lysate from each well was then added to $1 \mathrm{~mL}$ of nitric acid. The mixture samples were left at room temperature for $12 \mathrm{~h}$, followed by annealing at $80{ }^{\circ} \mathrm{C}$ for $6 \mathrm{~h}$ to remove the acid, and finally resuspended in DI water for Fe content determination by using ICP-AES (Optima 5300DV, PE, USA).

In vitro neutrophils recognition and endocytosis. Mouse neutrophils, treated with or without $10 \mu \mathrm{M}$ fMLF (Sigma, St. Louis) for 10 minutes at $37^{\circ} \mathrm{C}$, were incubated with $100 \mu \mathrm{g} / \mathrm{ml}$ of rhodamine-labeled TNPs and PTNPs for $30 \mathrm{~min}$. The cells were fixed and visualized under CLSM (LSM 880, Zeiss) and further analyzed by flow cytometry (AccuriC6, BD, USA). For visualization of Fe, the cells were stained with Prussian Blue. To validate the biomimetic function of PSGL-1, neutrophils were pretreated with anti-PSGL-1 monoclonal antibody (BioXcell, West Lebanon, NH) to block the PSGL-1, which was termed as the blocked PSGL-1 group. 
In vitro cytotoxicity assay. Neutrophils were pre-stimulated with $10 \mu \mathrm{M}$ fMLF for 10 minutes at $37{ }^{\circ} \mathrm{C}$. After washing, cells $\left(1 \times 10^{4}\right.$ cells/well $)$ were seeded in 96-well plates and cultured in the FBS free medium for 30 min. Afterward, neutrophils were incubated with different concentrations (i.e., 12.5, 25, 50, 100, 200, 400 and 800 $\mu \mathrm{g} / \mathrm{ml}$ ) of TNPs and PTNPs for $1 \mathrm{~h}$, followed by adding $5 \mathrm{mg} / \mathrm{mL}$ CCK-8 solution. After $4 \mathrm{~h}$ of incubation, the absorbance was measured at the wavelength of $450 \mathrm{~nm}$ by a microplate reader. Cells grown without any treatment were used as control.

Real-time fluorescence intravital microscopy. In vivo intravital microscopy of the cremaster muscle was performed as reported. ${ }^{6} \mathrm{TNF}-\alpha(0.5 \mu \mathrm{g}$ in $250 \mathrm{~mL}$ saline $)$ (R\&D Systems, Minneapolis, MN) was intrascrotally injected into male mouse (6-8 weeks old, C57BL/6). At 2-2.5 h after TNF- $\alpha$ injection, mice were anaesthetized by intraperitoneal injection of $4 \%$ Chloral hydrate $(0.1 \mathrm{ml} / 10 \mathrm{~g})$ and placed on a thermo-controlled blanket at $37^{\circ} \mathrm{C}$ on an intravital microscope tray. After excision of the scrotum, the cremaster muscles were incised vertically and flattened over a glass coverslip by pinning the periphery. To maintain tissue integrity throughout the experiment, pre-warmed buffer was superfused over the surgical field exteriorized on the intravital microscope table. Cy5-loaded NPs $(100 \mu \mathrm{g})$ in $100 \mu \mathrm{L}$ saline were intravenously injected, followed by injection of the Alexa Fluor 488-labeled anti-Gr-1 antibody $(0.05 \mu \mathrm{g} / \mathrm{g}$ BW in $100 \mu \mathrm{L}$ saline $)$. For in vivo blocking of PSGL-1, $50 \mu \mathrm{g}$ anti-PSGL-1 antibody were intravenously injected. Neutrophil rolling and adhesion 
were visualized using a $\times 20 / 1.0$ NA water immersion objective lens and a high-speed camera (Zeiss LSM 880, Germany).

In vivo neutrophils recognition in ischemic region. Male $\mathrm{C} 57 \mathrm{BL} / 6$ mice with ischemic stroke were used for in vivo recognition. Cy5-loaded TNPs and PTNPs at Cy5 dose of $20 \mathrm{nmol} / \mathrm{kg}$ were intravenously injected at $24 \mathrm{~h}$ post-reperfusion of MCAO model. For in vivo blocking of PSGL-1, $50 \mu \mathrm{g}$ anti-PSGL-1 antibody were intravenously injected. $4 \mathrm{~h}$ after injection, the mice were sacrificed. The brain tissues were dissected for ex vivo fluorescence imaging by using in vivo imaging system (PerkinElmer, USA). The fluorescence intensities of region-of-interests (ROI) were analyzed by Living Image Software.

To study in vivo discerning ability of PTNPs to adherent neutrophils. Cy5-loaded PTNPs were intravenously administered to tMCAO mice $24 \mathrm{~h}$ after reperfusion. Approximately $4 \mathrm{~h}$ later, mice were sacrificed. The brains were removed without perfusion and fixed in $4 \%$ formaldehyde for $4 \mathrm{~h}$, followed by dehydrating in $15 \%$ sucrose and then in $30 \%$ sucrose. Brains were frozen with O.C.T at $-80{ }^{\circ} \mathrm{C}$ and coronal series sections $(30 \mu \mathrm{m})$ were cut on a freezing microtome (Leica CM1520, Germany). Sections were blocked with $10 \%$ goat serum at room temperature for $1 \mathrm{~h}$, and then incubated with anti-Ly6g antibody (Abcam, Cambridge, MA) at $4{ }^{\circ} \mathrm{C}$ overnight. Isotype matched $\mathrm{IgG}$ is the control. After washing, brain sections were incubated with secondary antibody Alexa FlourTM 568 (Thermo Fisher Scientific, 
San Jose, CA) for $1 \mathrm{~h}$. Brain sections were examined using a confocal microscope (LSM 880, Zeiss, Germany).

Syk phosphorylation. Isolated mouse neutrophils $\left(2 \times 10^{6}\right.$ cells $)$ were seeded in 6-well plate and incubated with TNPs or PTNPs $(200 \mu \mathrm{M}$ as piceatannol $)$ at $37^{\circ} \mathrm{C}$ in the presence of $50 \mathrm{ng} / \mathrm{ml} \mathrm{TNF- \alpha}$ for 30 minutes. Non-adherent and adherent neutrophils were collected and lysed with Mammalian Protein Extraction Reagent (proteinase inhibitor cocktail, $1 \mathrm{mM}$ PMSF). The samples containing equivalent total proteins were prepared in lithium dodecyl sulfate (LDS) sample loading buffer and separated on $15 \%$ of bis-tris-polyacryl-amide gel. The gel was transferred onto a PVDF membrane and immunoblotted with an anti-phosphoSyk-Tyr525/526 antibody (absin, China).

Adhesion and transmigration assay. For adhesion assay, $2 \times 10^{5}$ HUVECs were seeded in 6-well plate. After $12 \mathrm{~h}$, HUVECs were stimulated with $10 \mathrm{ng} / \mathrm{ml} \mathrm{TNF-} \alpha$ for $4 \mathrm{~h}$ at $37{ }^{\circ} \mathrm{C}$. The isolated neutrophils were pre-stimulated with $10 \mu \mathrm{M}$ fMLF for 10 minutes at $37{ }^{\circ} \mathrm{C}$ and then treated with blank medium, TNPs, PTNPs for $30 \mathrm{~min}$, respectively. $1 \times 10^{6}$ treated neutrophils were seeded on top of the endothelial monolayer. After $15 \mathrm{~min}$, non-adherent cells were washed out and cells were stained with Alexa Fluor 488-labeled anti-Gr-1 antibody, followed by visualization using CLSM (LSM 880, Zeiss) and measurement using flow cytometry (AccuriC6, BD, USA). 
For transmigration assay, $2 \times 10^{5}$ HUVECs were seeded on $3 \mu \mathrm{m}$ polycarbonate membrane, then placed into 6-well trays and cultured for 3 or 4 days at $37{ }^{\circ} \mathrm{C}$. Prior to the experiment, HUVECs were stimulated with $10 \mathrm{ng} / \mathrm{ml} \mathrm{TNF}-\alpha$ for $4 \mathrm{~h}$ at $37{ }^{\circ} \mathrm{C}$. $1 \times 10^{6}$ treated neutrophils were seeded on top of the endothelial monolayer. After $1 \mathrm{~h}$ at $37^{\circ} \mathrm{C}$, transmigrated cells were collected from the bottom chambers and from the lower side of the filter, counted by using a Coulter counter, and the counts were expressed as a percentage of the total number of neutrophils added.

In vivo neutrophils monitoring by MRI. To study the MRI imaging of neutrophils infiltration into tMCAO mice, $200 \mu \mathrm{L}$ of TNPs and PTNPs $(\sim 15 \mathrm{mg}$ iron per $\mathrm{kg}, 2$ mg piceatannol per $\mathrm{kg}, \mathrm{n}=3$ per group) were intravenously injected on hours 6,12 and 18 after reperfusion. To determine the selectivity of PTNPs, a further group of mice $(\mathrm{n}=3)$ were intravenously injected with $50 \mu \mathrm{g}$ anti-PSGL-1 antibody (clone 4RA10, bxcell, USA) to block PSGL-1-binding sites at $15 \mathrm{~min}$ before administration of PTNPs. $4 \mathrm{~h}$ after PTNPs injection, mice were placed in a quadrature birdcage coil with an in-built stereotaxic frame for imaging on a 7.0 $\mathrm{T}$ whole body MRI system (Biospec 7T/20 USR, Bruker, Germany). The imaging protocol consisted of $T_{2}$ measurement using a TurboRARE sequence (TR/TE: $2500 / 33 \mathrm{~ms}$, flip angle $180^{\circ}$ ), and a fast low angle shot (FLASH) $T_{2}{ }^{*}$-weighted sequence (TR/TE: $400 / 3.5 \mathrm{~ms}$, flip angle $30^{\circ}$ ). For each magnetic resonance image, we manually masked the brain to exclude extracerebral structures. ImageJ freeware was used to segment and to quantify the number of dark voxels in ischemic region. ${ }^{7}$ 
In vivo therapeutic efficacy assay. $200 \mu \mathrm{L}$ of saline, TNPs and PTNPs $(\sim 15 \mathrm{mg}$ iron per $\mathrm{kg}, 2 \mathrm{mg}$ piceatannol per $\mathrm{kg}$ ) were intravenously injected into tMCAO mice on hours 6,12 and 18 after reperfusion. Approximately $24 \mathrm{~h}$ after reperfusion, mice were sacrificed and the brains were harvested. For neutrophil infiltration study, immunofluorescence staining was performed. Neutrophils were labeled with anti-Ly6g antibody (Abcam, Cambridge, MA), and DAPI was used for nuclear staining. The distribution of fluorescence was visualized under a confocal microscope (LSM 880, Zeiss). For measurement of infarct volume rate, TTC staining was performed. Briefly, the brains were cut into 5 slices ( $2 \mathrm{~mm}$ thickness), then stained with $2 \%$ TTC at $37{ }^{\circ} \mathrm{C}$ for $10 \mathrm{~min}$. All TTC-stained slices of each brain were photographed with a digital camera. The infarcted area in each section was measured using an Image-ProPlus analysis system. Briefly, infarction volumes were determined following the formula: $(\mathrm{CH}-\mathrm{NLH}) / \mathrm{CH} \times 100 \%$. NLH indicates the area of non-lesioned ipsilateral hemisphere, $\mathrm{CH}$ indicates the area of the contralateral hemisphere $(\mathrm{CH})$.

For inflammatory cytokines and MPO activity measurement, infarcted half brains were collected and homogenized. Supernatant was collected and detected for MPO activity, TNF- $\alpha$ and IL-1 $\beta$ level by corresponding ELISA kits. Experiments were conducted according to the protocol in triplicate. Neurological deficits were measured on a five-point scale. No neurological deficit $=0$, failure to extend right paw fully $=1$, 
circling to right $=2$, falling to right $=3$, mice did not walk spontaneously and had depressed levels of consciousness $=4$.

Statistical Analysis. All the data were presented as mean \pm SD. Student's t-test was used for a comparison between two groups, and the differences among multiple groups were analyzed by one-way ANOVA. Statistical significance was determined with a $\mathrm{P}$ value lower than 0.05 .

\section{References}

(1) Rousselet, E.; Kriz, J.; Seidah, N. G. Jove-J Vis Exp 2012, (69).

(2) Xu, Z. C.; Shen, C. M.; Hou, Y. L.; Gao, H. J.; Sun, S. S. Chem Mater 2009, 21, (9), 1778-1780.

(3) Kim, J.; Lee, J. E.; Lee, S. H.; Yu, J. H.; Lee, J. H.; Park, T. G.; Hyeon, T. Advanced Materials 2008, 20, (3), 478-483.

(4) Hu, C. M.; Fang, R. H.; Wang, K. C.; Luk, B. T.; Thamphiwatana, S.; Dehaini, D.; Nguyen, P.; Angsantikul, P.; Wen, C. H.; Kroll, A. V.; Carpenter, C.; Ramesh, M.; Qu, V.; Patel, S. H.; Zhu, J.; Shi, W.; Hofman, F. M.; Chen, T. C.; Gao, W.; Zhang, K.; Chien, S.; Zhang, L. Nature 2015, 526, (7571), 118-121.

(5) Xue, J. W.; Zhao, Z. K.; Zhang, L.; Xue, L. J.; Shen, S. Y.; Wen, Y. J.; Wei, Z. Y.; Wang, L.; Kong, L. Y.; Sun, H. B.; Ping, Q. N.; Mo, R.; Zhang, C. Nature Nanotechnology 2017, 12, (7), 692-700. 
(6) Kim, K. H.; Barazia, A.; Cho, J. Jove-J Vis Exp 2013, (74).

(7) Farr, T. D.; Lai, C. H.; Grunstein, D.; Orts-Gil, G.; Wang, C. C.; Boehm-Sturm, P.; Seeberger, P. H.; Harms, C. Nano Letters 2014, 14, (4), 2130-2134. 

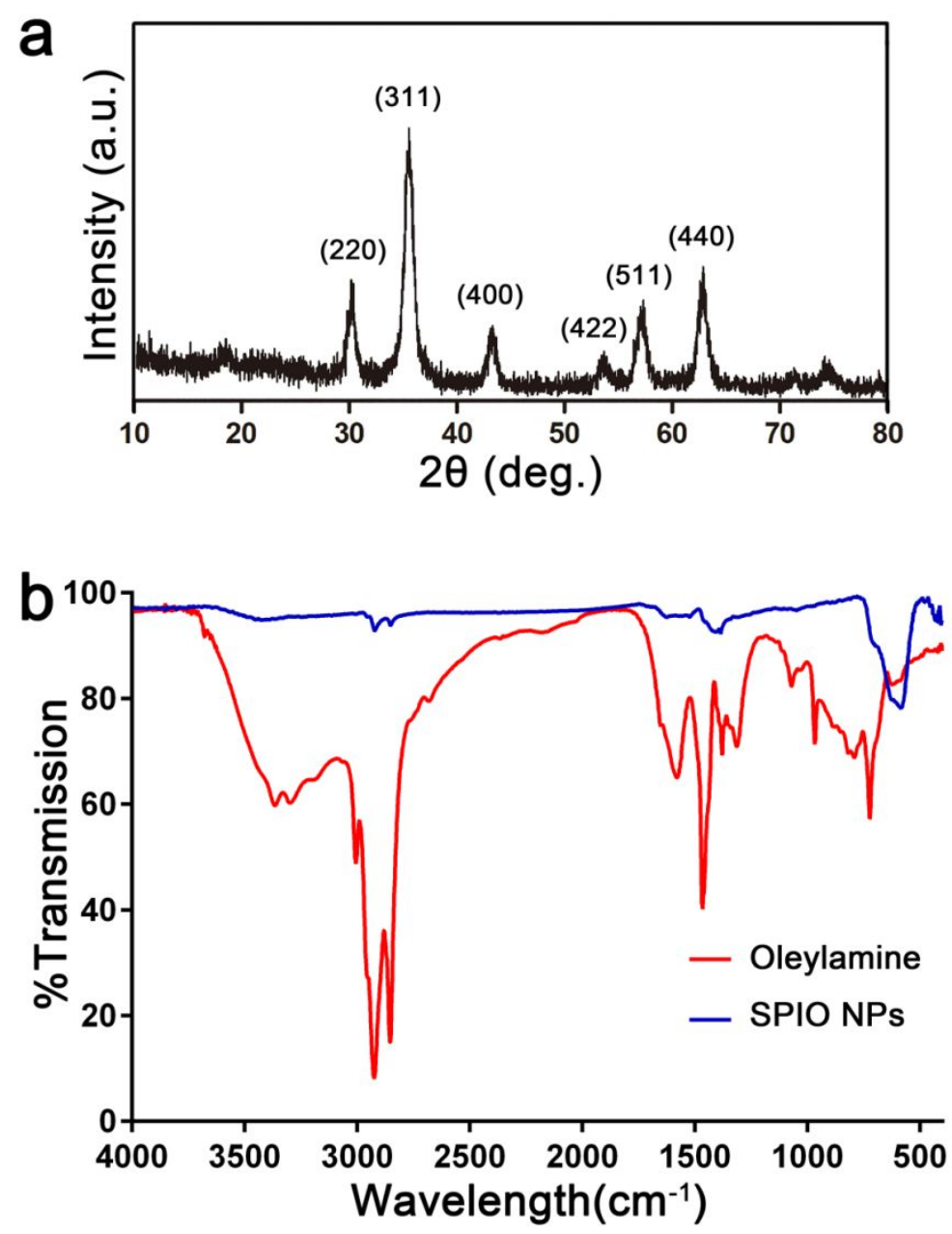

Figure S1. a) X-ray diffraction pattern and b) FT-IR spectra of SPIO nanoparticles. 


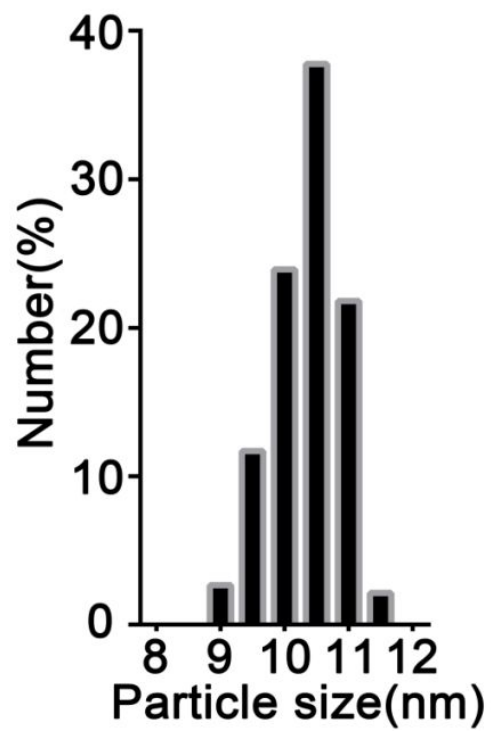

Figure S2. The size distribution histograms of SPIO nanoparticles. 


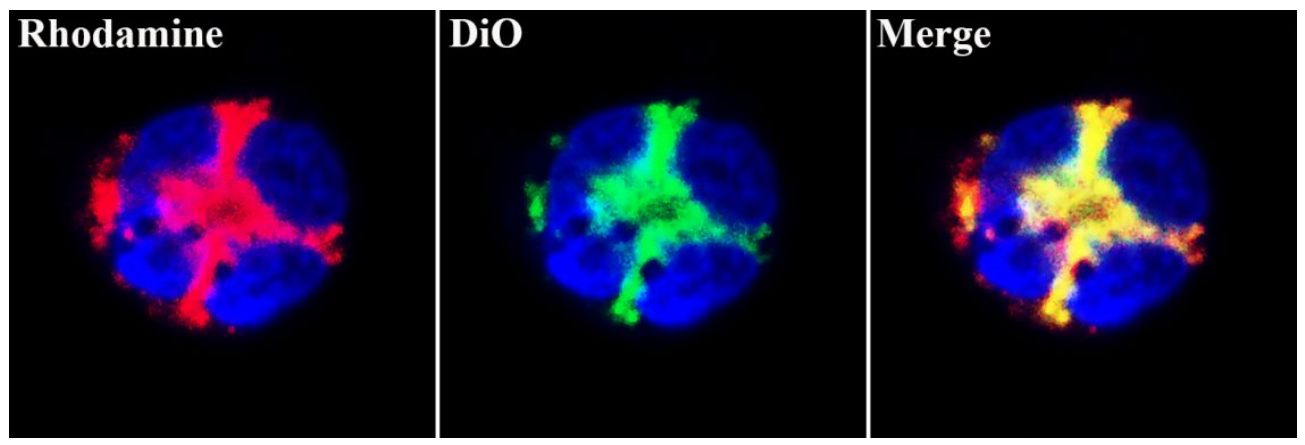

Figure S3. Representative CLSM images of single neutrophil after incubation with

PTNPs. Cell nucleus, PLGA core, and platelet membrane were labeled with Hoechst 33342 (blue), Rhodamine (red), and $\mathrm{DiO}$ (green), respectively. 

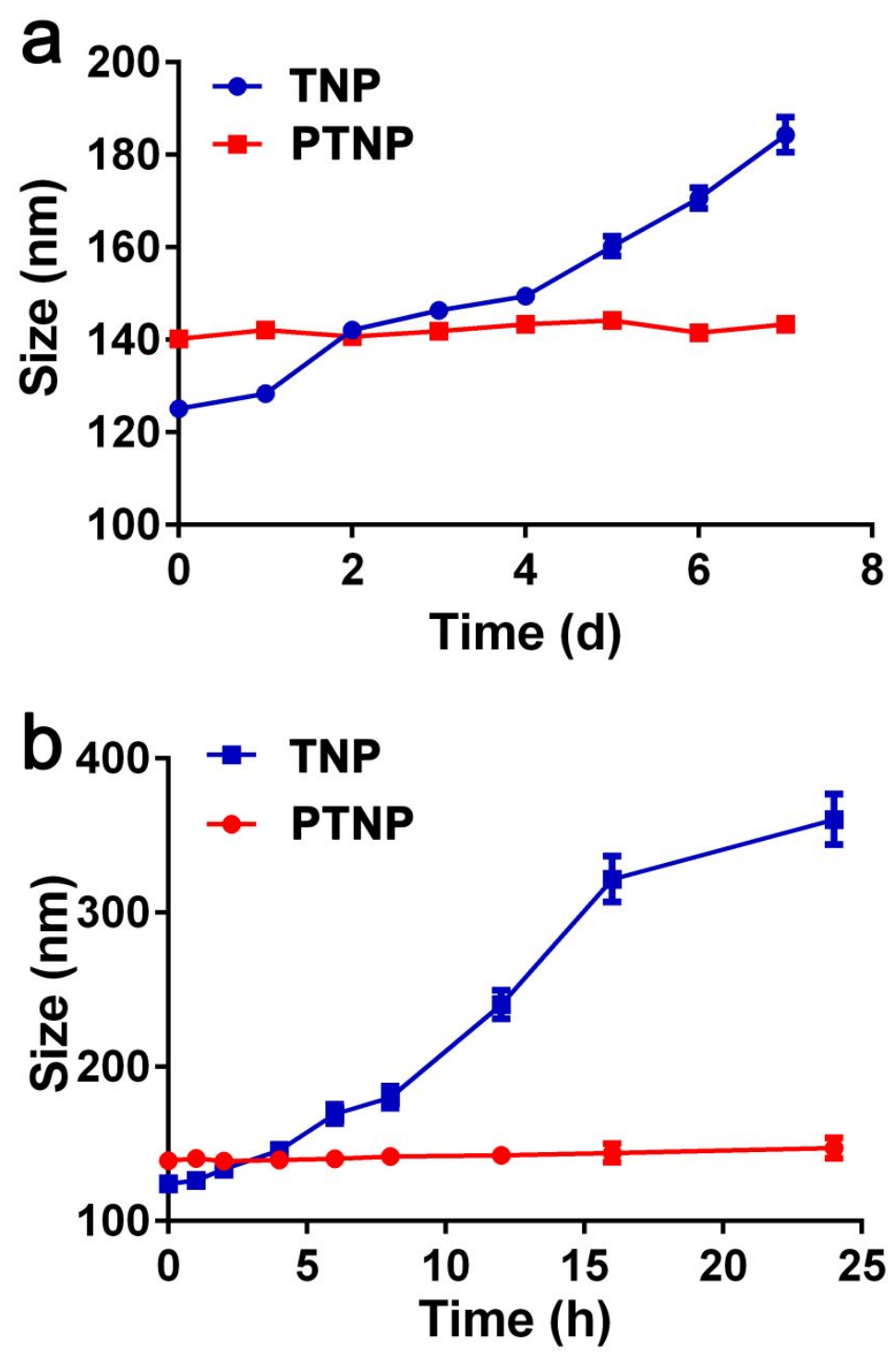

Figure S4. a) PBS and b) $100 \%$ FBS stability of TNP and PTNP monitored by dynamic light scattering at $37^{\circ} \mathrm{C}$. All values are expressed as mean $\pm \mathrm{SD}(\mathrm{n}=3)$. 


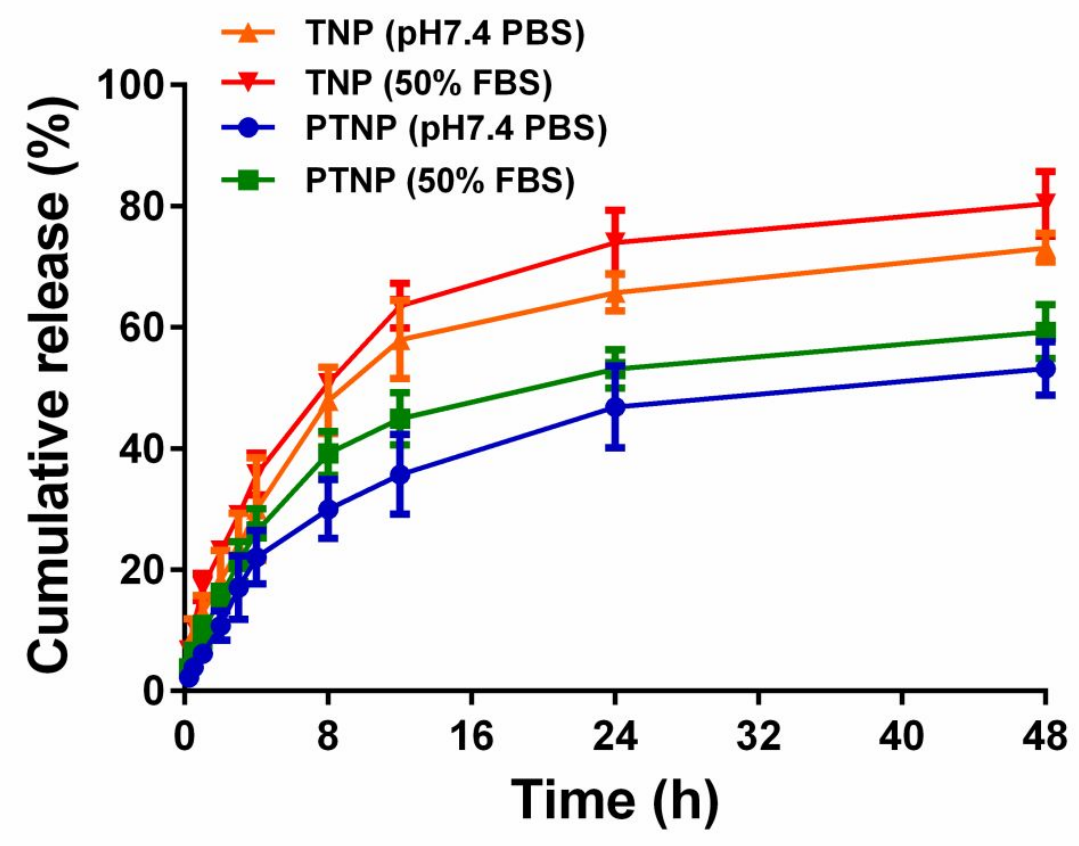

Figure S5. In vitro release of piceatannol from TNPs and PTNPs in pH7.4 PBS or $50 \%$ fetal bovine serum (FBS). All values are expressed as mean $\pm \operatorname{SD}(n=3)$. 


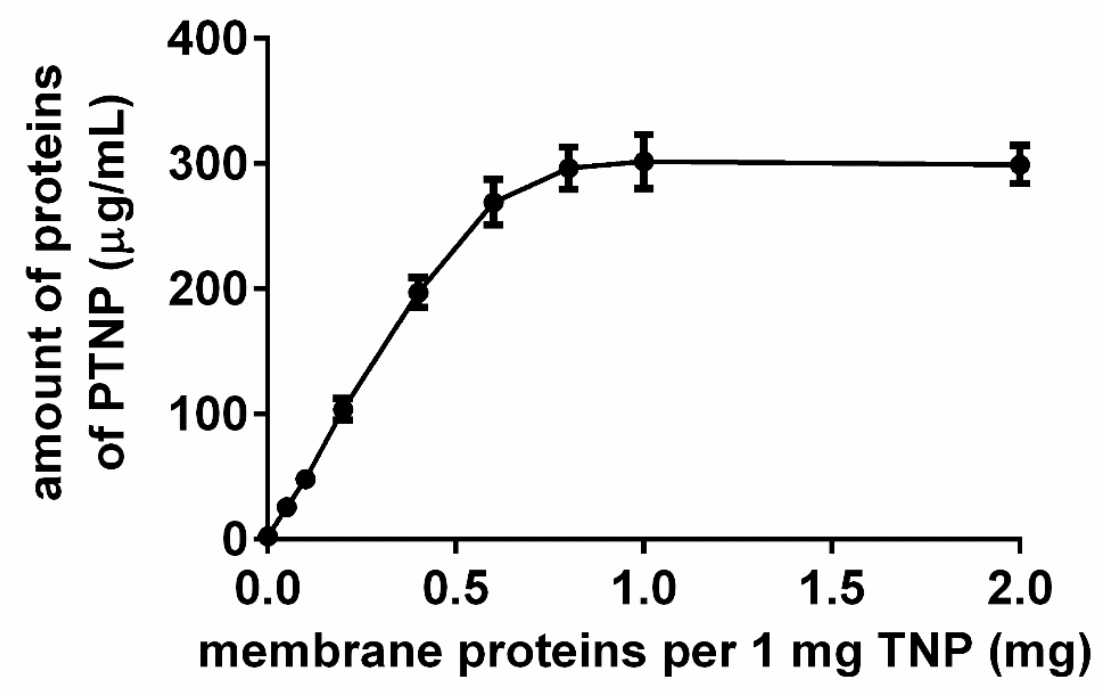

Figure S6. Quantification of total membrane proteins on PTNP by BCA assay after incubating different weight ratio of platelet membrane to TNP core from 0.05 to 2 $(n=3)$. 


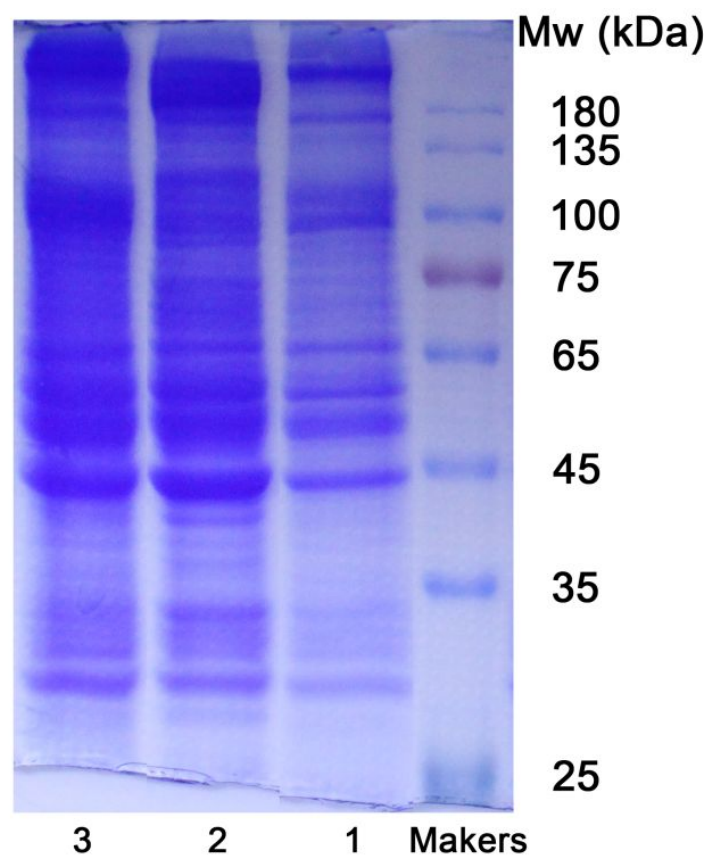

Figure S7. SDS-PAGE analysis of 1) platelet, 2) platelet vesicle and 3) PTNP. 


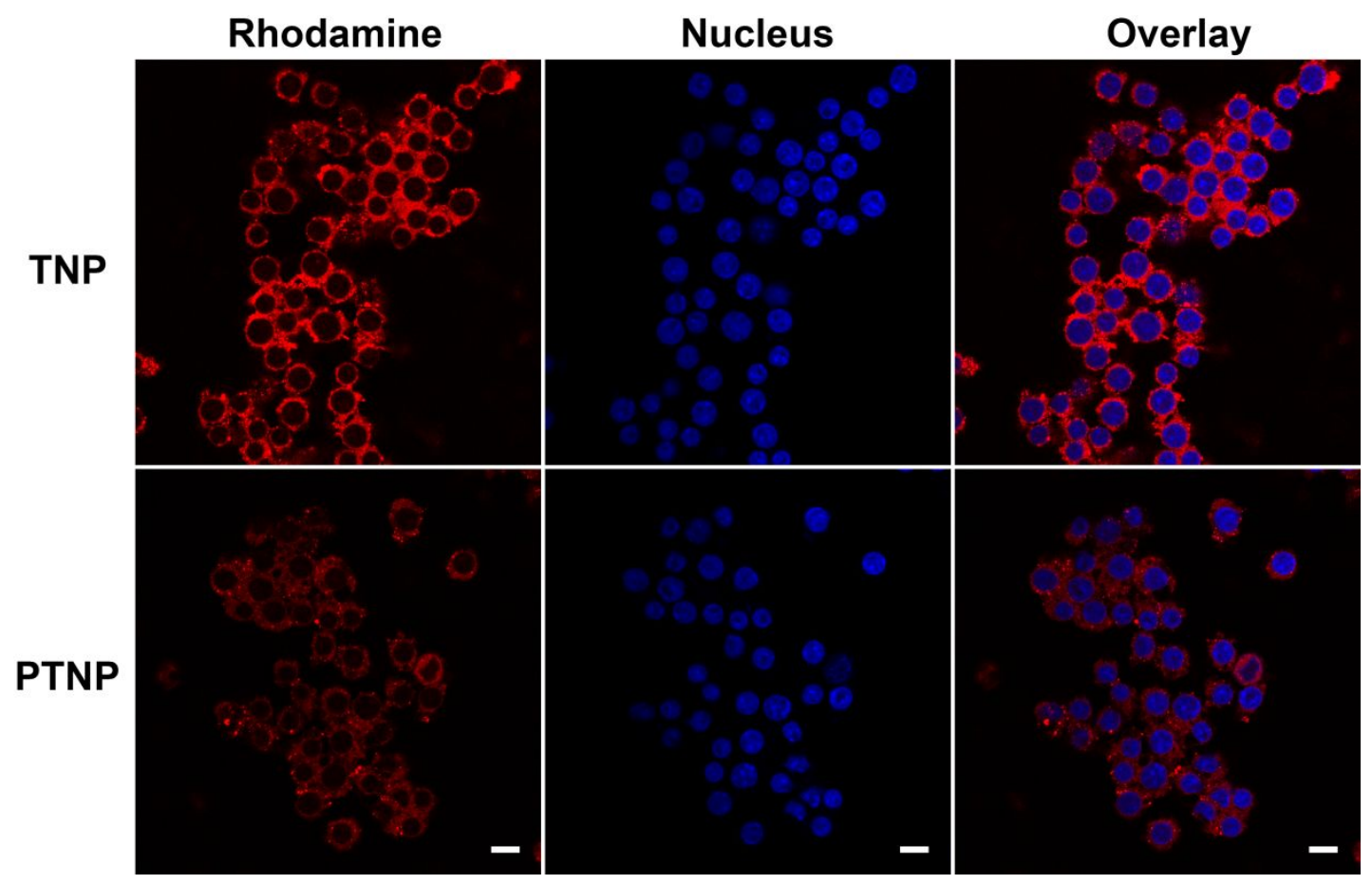

Figure S8. Intracellular uptake of TNPs and PTNPs in RAW264.7 cells after $1 \mathrm{~h}$ incubation. The nucleus was stained with Hoechst 33342 (blue). The TNPs and PTNPs were labeled with rhodamine (red). Scale bars, $5 \mu \mathrm{m}$. 


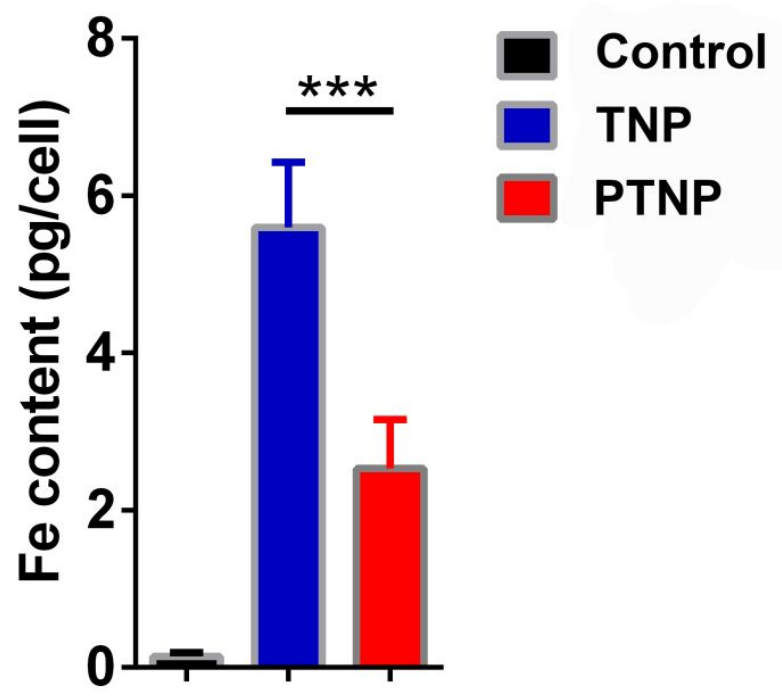

Figure S9. Quantitative analysis of the macrophage uptake of TNPs and PTNPs at $100 \mu \mathrm{g} \mathrm{Fe} \mathrm{mL} \mathrm{m}^{-1}$ concentration concentrations for $4 \mathrm{~h}$ incubation. All values are expressed as mean $\pm \mathrm{SD}(\mathrm{n}=6) .{ }^{* * *} P<0.001$. 

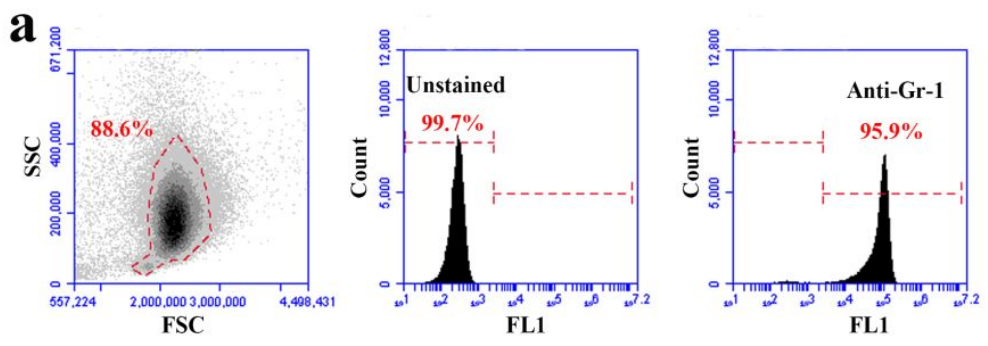

b
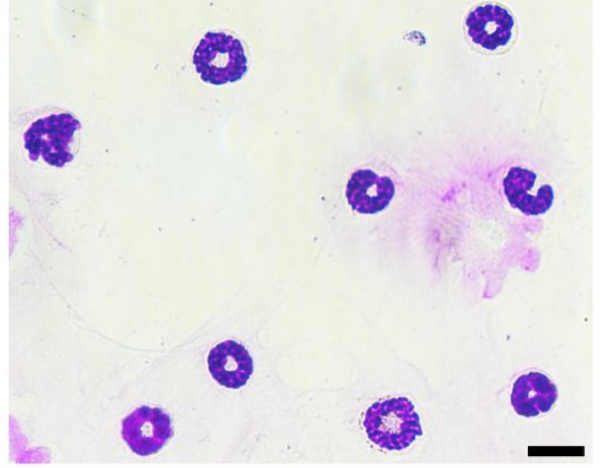

Figure S10. a) Flow cytometric analysis of the purity of neutrophils stained with FITC-conjugated Gr-1. b) Morphological images of neutrophils stained with Wright-Giemsa. Scale bars, $10 \mu \mathrm{m}$. 
$\square$ TNP $\square$ PTNP

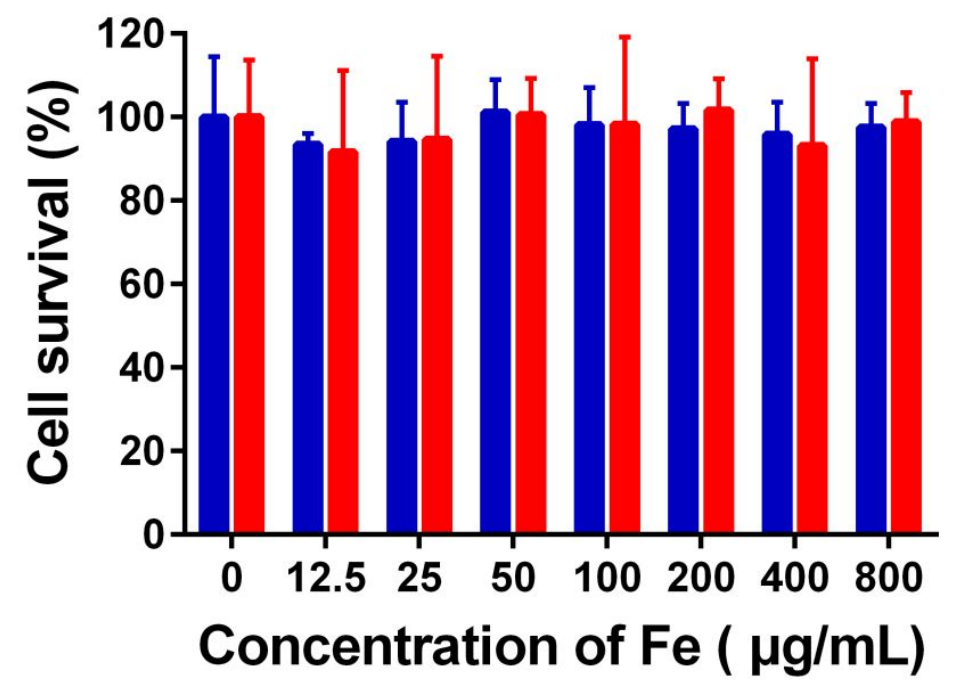

Figure S11. Neutrophils viability after incubation with TNPs and PTNPs at different concentrations. All values are expressed as mean $\pm \operatorname{SD}(n=6)$. 

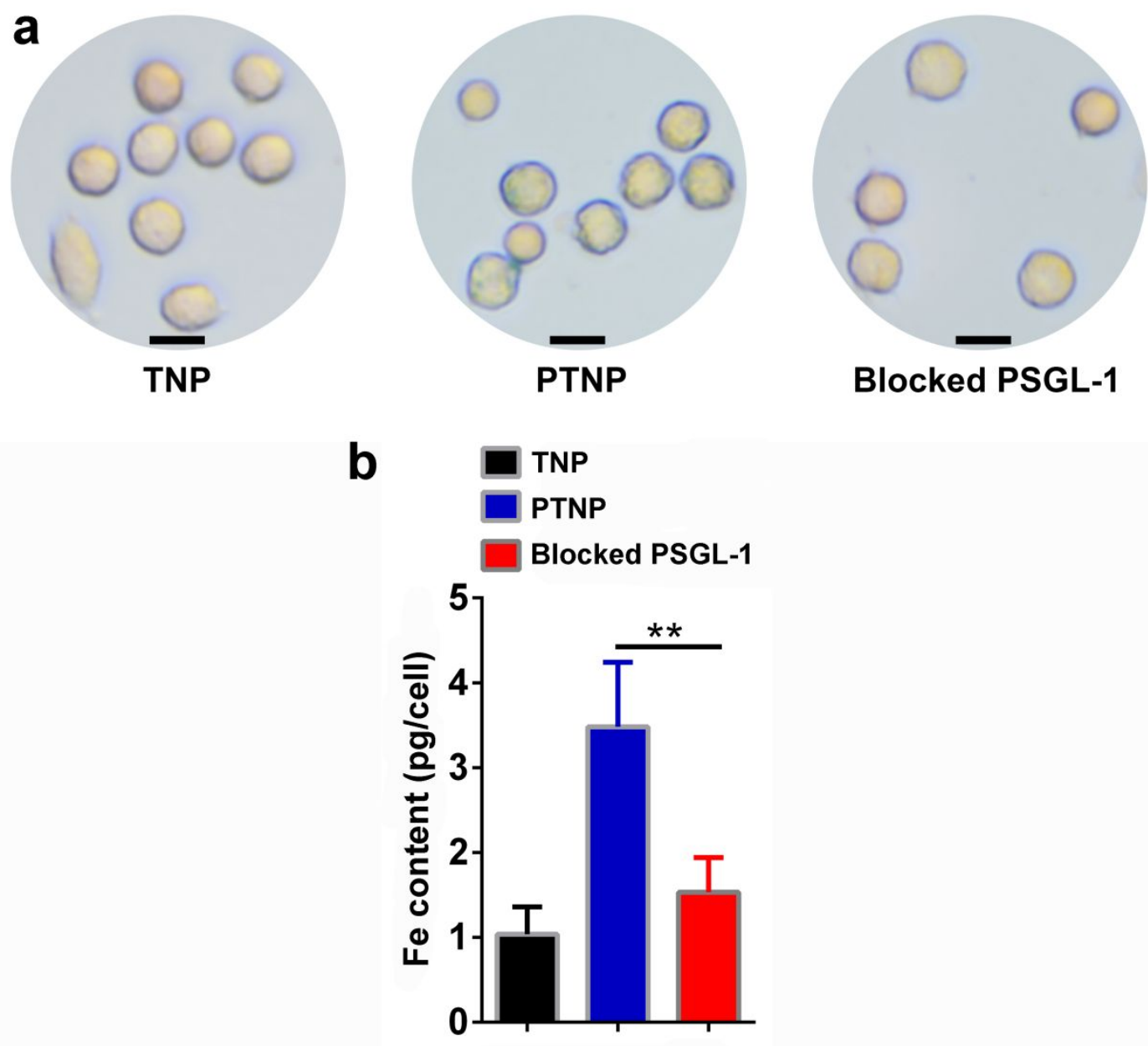

Figure S12. a) The cellular uptake of TNPs and PTNPs in mouse neutrophils, prestimulated with $10 \mu \mathrm{M}$ fMLF, determined by Prussian Blue staining. Scale bar: 10 $\mu \mathrm{m}$. The neutrophils blocked with PSGL-1 were used as a control. b) Quantitative analysis of uptake of various nanoparticles. Error bars indicate s.d. $(\mathrm{n}=3)$. ${ }^{* *} \mathrm{P}<$ 0.01 . 

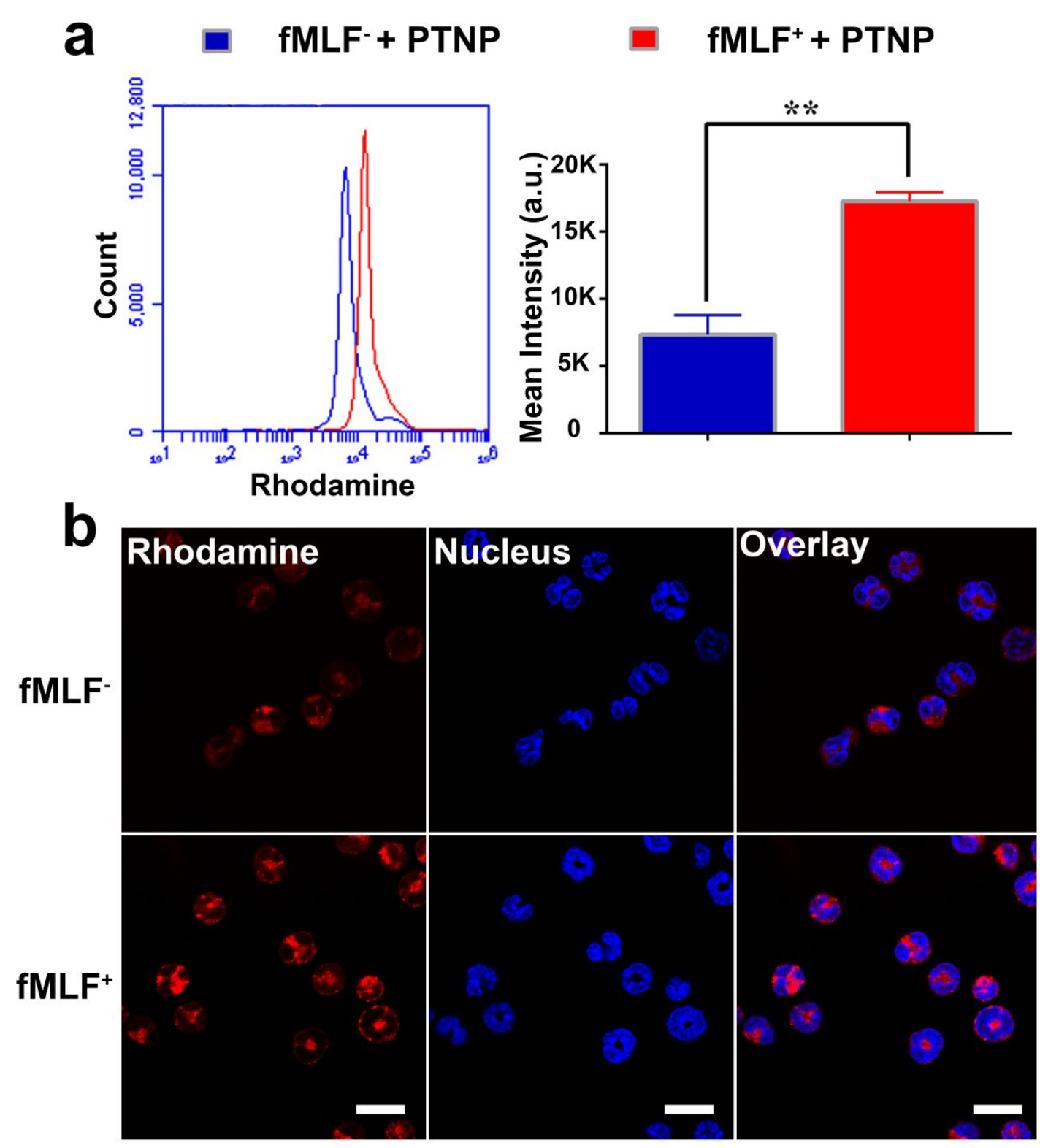

Figure S13. The cellular uptake of Rhodamine labeled PTNP in mouse neutrophils, pre-stimulated with or without $10 \mu \mathrm{M}$ fMLF, determined by FACS analysis (a) and CLSM (b) (n=3). Scale bar: $10 \mu \mathrm{m}$. 


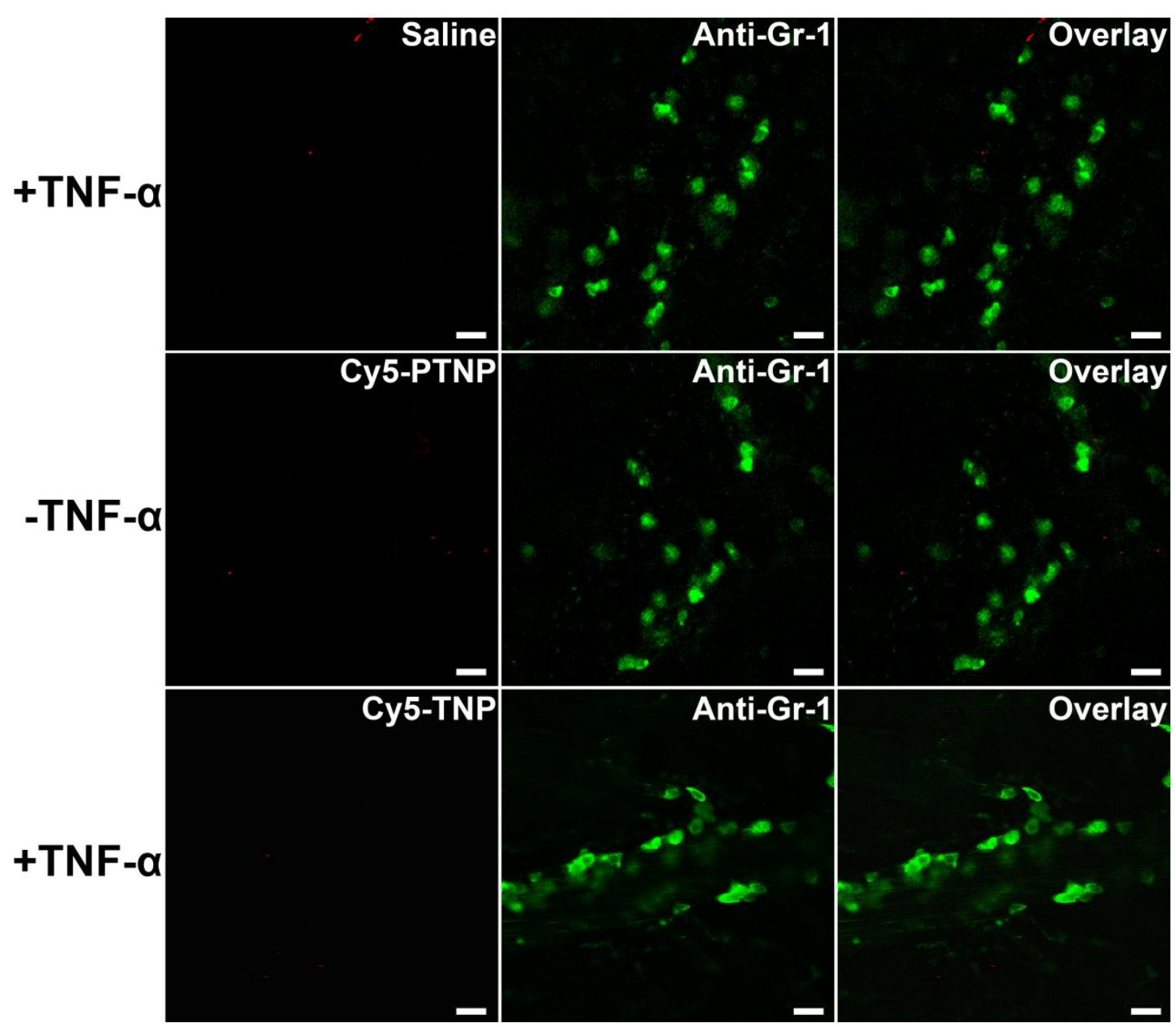

Figure S14. Intravital microscopy of neutrophils in cremaster muscle venules of mice by an intravenous infusion of saline, Cy5-loaded TNP and Cy5-loaded PTNP following intrascrotal injection of TNF-a or not. Scale bar: $20 \mu \mathrm{m}$. 

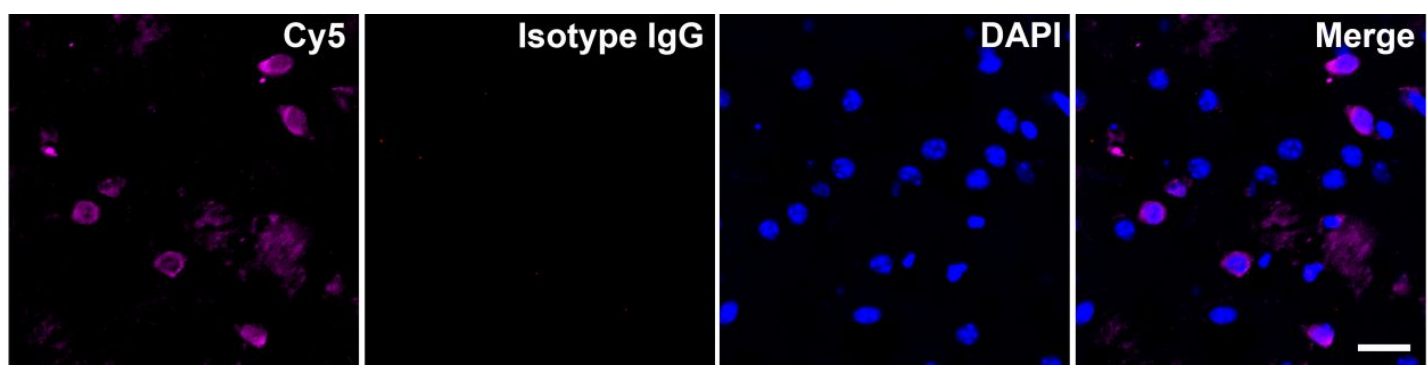

Figure S15. Specificity of anti-Ly6g antibody in mouse neutrophils. Isotype matched IgG is the control. Scale bar: $20 \mu \mathrm{m}$. 


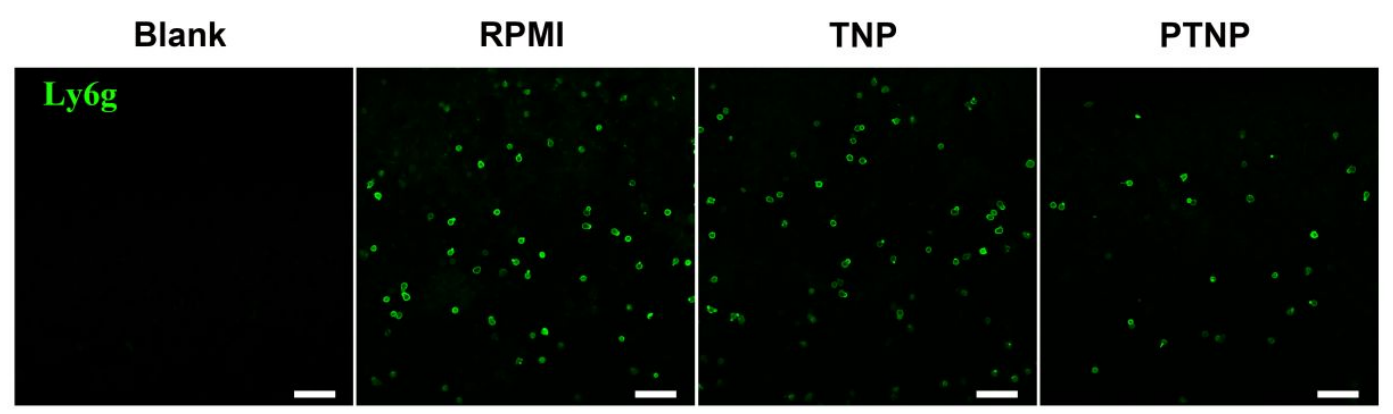

Figure S16. Evaluation of neutrophil adhesion to a HUVEC monolayer after incubation with TNPs and PTNPs (piceatannol, $200 \mu \mathrm{M}$ ). The culture medium RPMI was used as a negative control. Tightly confluent monolayers of HUVECs were stimulated with $10 \mathrm{ng} / \mathrm{mL} \mathrm{TNF- \alpha}$ for $4 \mathrm{~h}$. The adherent neutrophils were labelled with Alexa Fluor 488-labeled anti-Gr-1 antibody (green). Scale bar $=50 \mu \mathrm{m}$. 


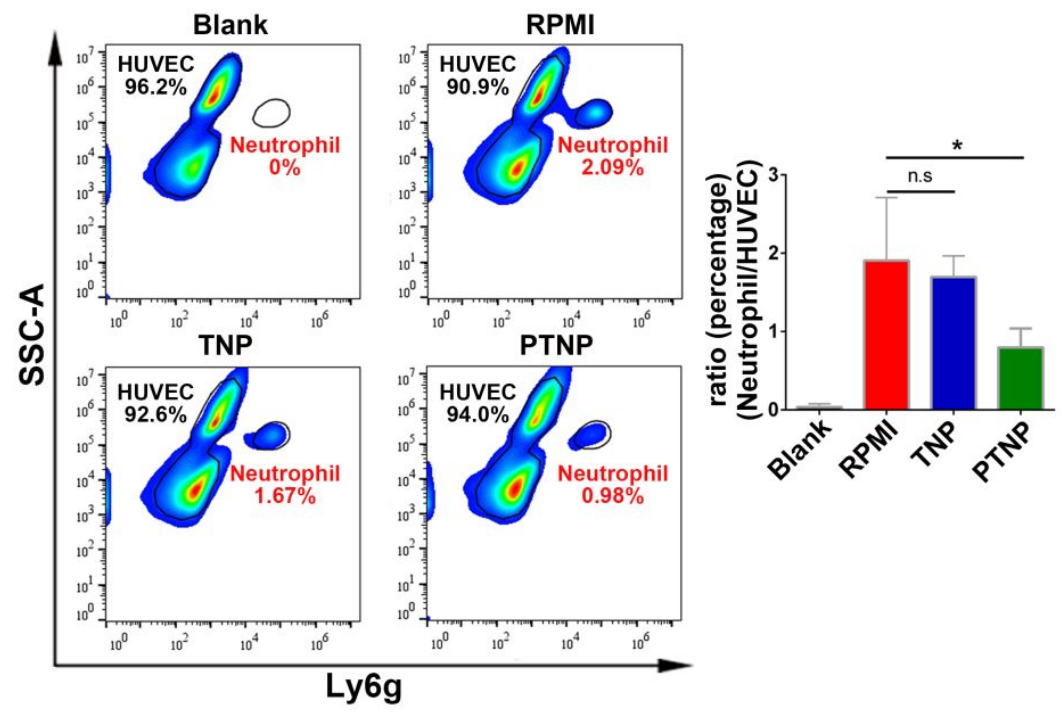

Figure S17. FACS analysis of neutrophil adhesion to HUVEC. All values are expressed as mean $\pm \mathrm{SD}(\mathrm{n}=3)$. ${ }^{*} P<0.05$. 

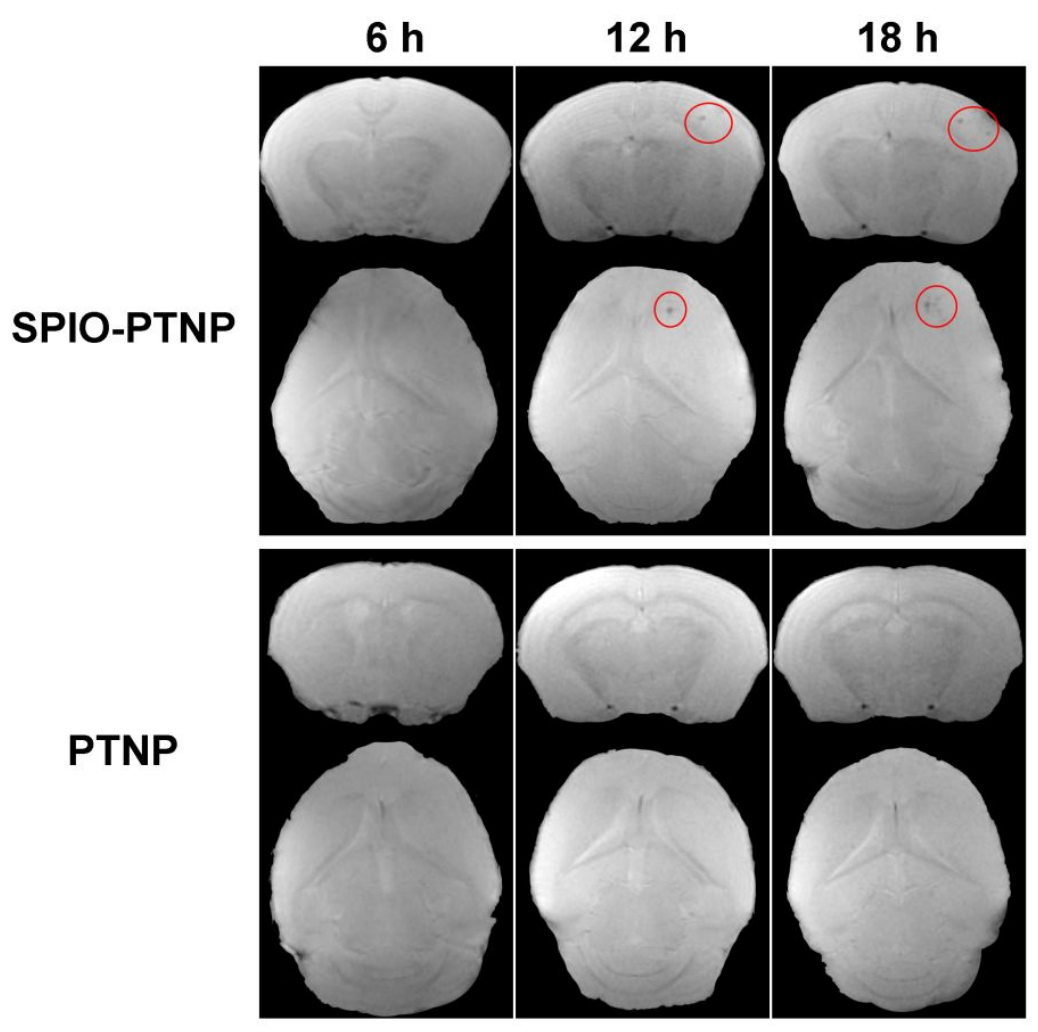

Figure S18. T2*-weighted coronal and transverse images at 6,12 , and $18 \mathrm{~h}$ after reperfusion of tMCAO mice just prior to SPIO-PTNPs or PTNPs administration. The little negative contrast in mice treated with SPIO-PTNPs at time $12 \mathrm{~h}$ and $18 \mathrm{~h}$, mainly due to the influence of the previous SPIO-PTNPs injection at time $6 \mathrm{~h}$ and 12 h. 


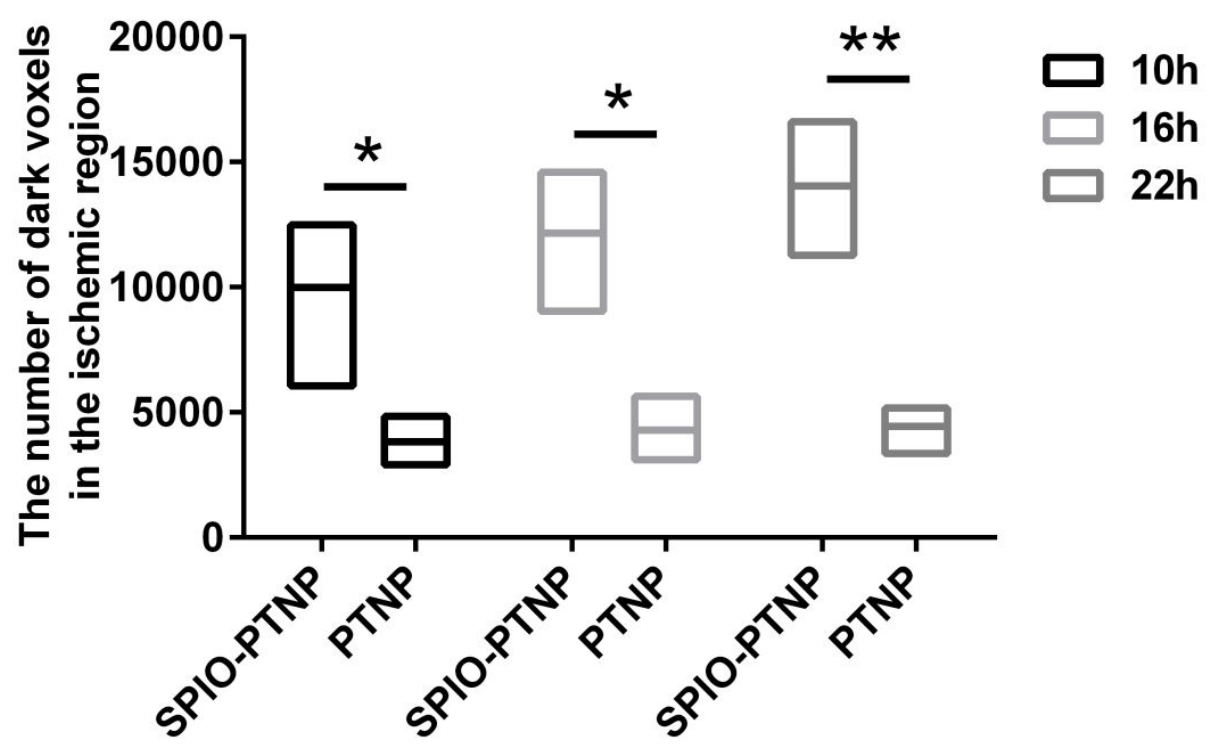

Figure S19. The total number of dark voxels in the ischemic region of the mice from groups SPIO-PTNPs and PTNPs $(\mathrm{n}=3) .{ }^{*} P<0.05, * * P<0.01$. 

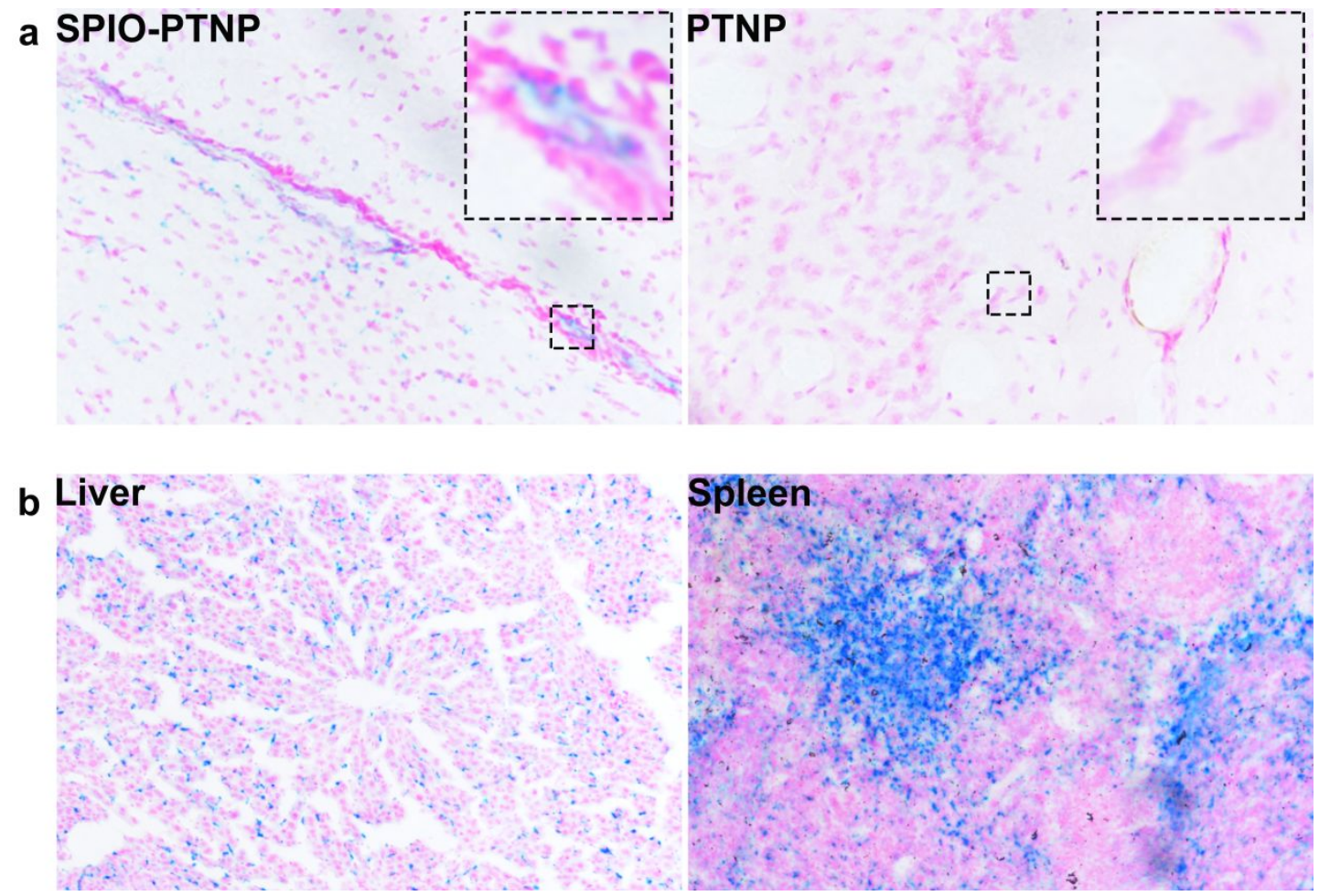

Figure S20. a) Prussian blue staining of ischemic brain section in the tMCAO mice treated with SPIO-PTNPs (without piceatannol loaded) and PTNPs $(40 \times)$. b) High amounts of iron were detected in the liver and spleen $(20 \times)$. 


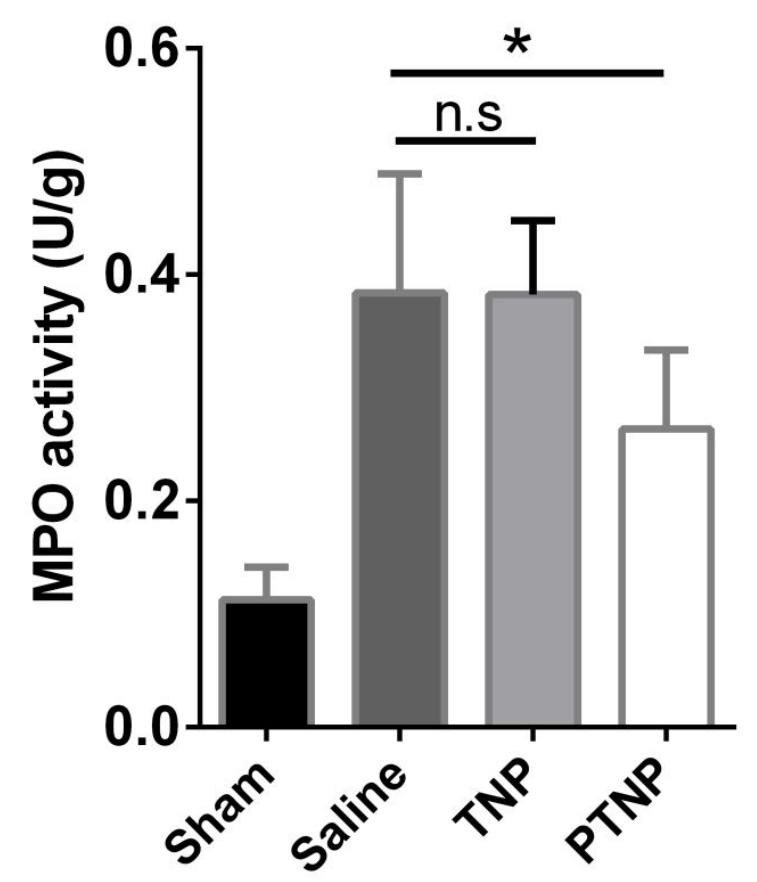

Figure S21. The myeloperoxidase (MPO) activity in the brains of tMCAO mice treated with saline, TNPs, and PTNPs. All values are expressed as mean \pm SD $(n=5)$. $* P<0.05$. 


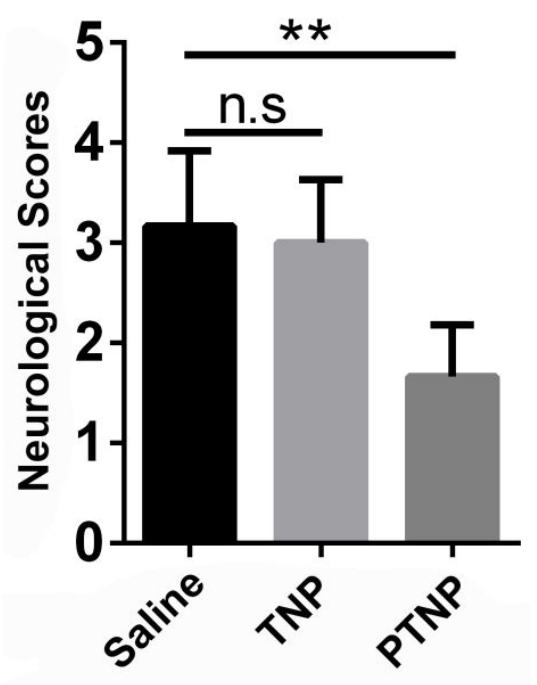

Figure S22. Effects of TNPs and PTNPs on neurological scores. All values are expressed as mean $\pm \mathrm{SD}(\mathrm{n}=5) . * * P<0.01$. 
Table S1. The content of SPIO, PLGA, Platelet membrane, and piceatannol in SPIO-TNPs, SPIO-PTNPs, TNPs and PTNPs.

\begin{tabular}{ccccc}
\hline Formulations & SPIO (\%) & PLGA (\%) & $\begin{array}{c}\text { platelet } \\
\text { membrane } \\
(\%)\end{array}$ & $\begin{array}{c}\text { piceatannol } \\
(\%)\end{array}$ \\
\hline SPIO-TNPs & 33.17 & 66.83 & 0 & 0 \\
SPIO-PTNPs & 31.89 & 64.26 & 0 & 3.85 \\
TNPs & 23.60 & 47.54 & 28.86 & 0 \\
PTNPs & 22.95 & 46.22 & 28.06 & 2.77 \\
\hline
\end{tabular}

